\title{
Covid-19 e infraestrutura hospitalar na Região do Araguaia Paraense
}

\section{Covid-19 and hospital infrastructure in Araguaia Paraense Region, Brazil}

Article Info:

Article history: Received 2021-03-29 / Accepted 2021-03-29 / Available online 2021-03-30

doi: $10.18540 /$ jcecvl7iss1pp12083-01-13e

Tarciso Binoti Simas

ORCID: https://orcid.org/0000-0002-1687-7582

Federal University of South and Southeast of Pará, Brazil

E-mail: tarcisobinoti@gmail.com

Ana Belén Cano Hila

ORCID: https://orcid.org/0000-0003-3145-9466

Barcelona University, Spain

E-mail: anabelencano@ub.edu

Carlos Maviael de Carvalho

ORCID: https://orcid.org/0000-0002-7777-4659

Federal University of South and Southeast of Pará, Brazil

E-mail: maviael.mcarvalho@gmail.com

\section{Resumo}

O novo coronavírus tem causado impactos negativos em todo mundo, sendo que cada região merece um olhar mais atento para suas particularidades. Assim, a proposta deste trabalho é analisar a evolução do Covid-19, parte das infraestruturas hospitalares e a hierarquia urbana nos municípios do Araguaia Paraense, localizada no Sudeste do Pará, Brasil. Esta pesquisa exploratória analisa os dados demográficos, de parte das infraestruturas de saúde e de casos do novo coronavírus registrados entre março e outubro de 2020 nos municípios da área de estudo correlacionando com os números estaduais e nacionais. Esta fotografia permite observar a carência de infraestruturas hospitalares em grande parte das cidades, indícios de subnotificação de casos e a relação de dependência entre alguns municípios. Além da perda de vidas humanas, esta crise sanitária escancara as assimetrias desta região periférica paraense e reforça a necessidade de ampliação das infraestruturas pelo Sistema único de Saúde (SUS).

Palavras-chave: Covid-19. Araguaia Paraense. Desenvolvimento urbano e regional.

\begin{abstract}
The new coronavirus has caused negative impacts worldwide and each region deserves a closer look at its particularities. Thus, the objective of this work is to investigate the evolution of Covid-19, part of the hospital infrastructure and the urban hierarchy in the municipalities of Araguaia Paraense, located in the Southeast of Pará, Brazil. This exploratory research analyzes demographic data, part of the health infrastructures and cases of new coronavirus recorded between March and October 2020 in the municipalities of study area, correlating with the state and national numbers. This photograph allows us to observe the lack of hospital infrastructure in most cities, evidence of underreporting of cases and the dependency relationship between some municipalities. In addition to the loss of human lives, this health crisis shows the asymmetries of this peripheral region of Pará and reinforces the need for expansion of infrastructures by the Unified Health System.
\end{abstract}

Keywords: Covid-19. Araguaia Paraense. Urban and regional development. 
O novo coronavírus tem causado muitos impactos negativos, sobretudo na vida humana. Até 02 de outubro de 2020, em todo mundo, mais de um milhão de mortes e 34 milhões de casos foram registrados, com os maiores impactos nas Américas com mais de 16 milhões de casos, 10 milhões de recuperados e 500 mil mortes (OPAS, 2020). No Brasil, em 04 de agosto, foram registrados mais de 4 milhões de casos e 140 mil óbitos (CB, 2020). Apesar da calamidade generalizada, cada região tem sido impactada de forma diferente e tem ressaltado suas particularidades. Assim, distinto das pesquisas realizadas nas principais centralidades do país, este trabalho investiga especificamente a Região do Araguaia Paraense ${ }^{1}$, situada na Mesorregião do Sudeste Paraense, entre os rios Araguaia e Xingu, e na fronteira com Mato Grosso (MT) e Tocantins (TO), conforme Figura 1.
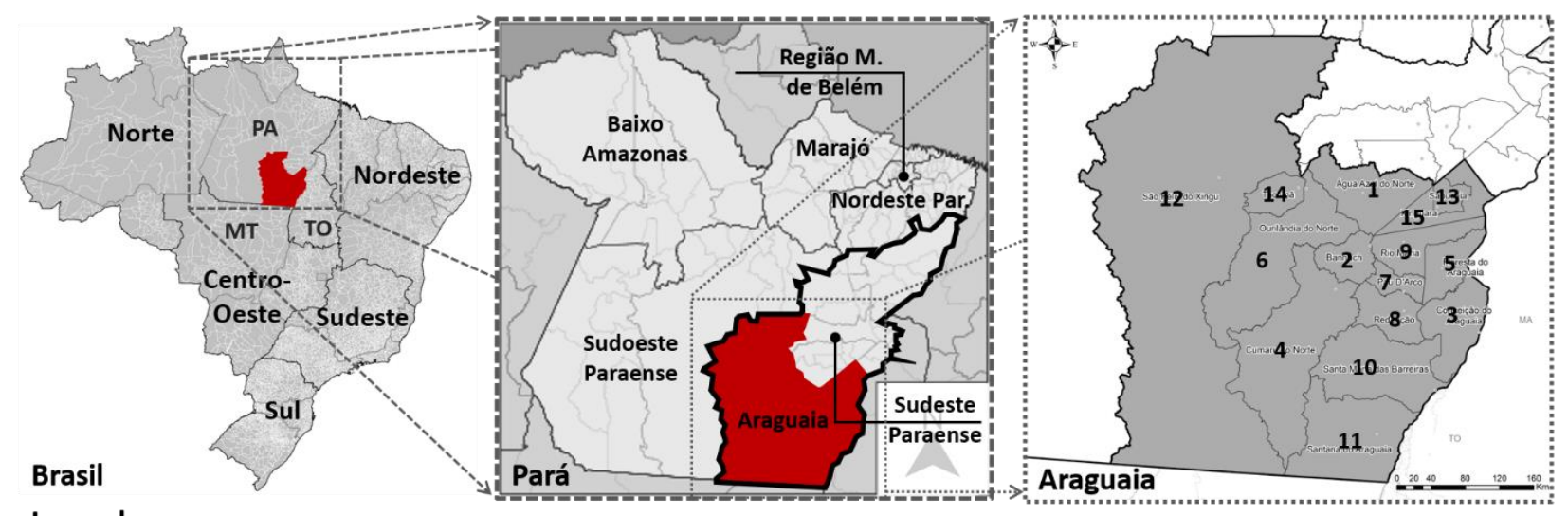

Legenda

Municípios: (1) Água Azul do Norte, (2) Bannach, (3) Conceição do Araguaia, (4) Cumaru do Norte, (5) Floresta do Araguaia, (6) Ourilândia do Norte, (7) Pau D’arco, (8) Redenção, (9) Rio Maria, (10) Santa Maria das Barreiras, (11) Santana do Araguaia, (12) São Félix do Xingu, (13) Sapucaia, (14) Tucumã e (15) Xinguara

Região de Integração Araguaia ou Região Geográfica Intermediária de Redenção

Figura 1 - Localização da Região do Araguaia e seus municípios

Fonte: Tarciso Simas (2020).

Silva (2007) destaca que, originalmente, essas terras e matas eram ocupadas por índios Kayapó, subdivididos em três grupos: Kradaú, ou Irã Aimrare, Gorotire e Kuben-Kran-Kegn. Eles tinham uma essência nômade e, por isso, constituíam diferentes aldeias na região. Com a ocupação não-indígena, o grupo Kradaús tornou-se extinto sendo parcialmente introduzido pela onda pastoril e atraído para os núcleos urbanos, enquanto os grupos Gorotire e Kuben-Kran-Kegn foram confinados em reservas indígenas ao longo do século XX. Segundo o autor, essa ocupação nãoindígena da região oriental da Amazônia Brasileira se deve, principalmente: (i) nos anos 1890, pela expansão da pecuária sertaneja de origem nordestina (sobretudo maranhense) e a ocupação na margem paraense do Rio Araguaia e em seus afluentes rios Pau D'Arco e Arraias; (ii) na primeira metade do século XX, pela extração do caucho, quando os povoados sertanejos serviam de pontos de apoio no transporte de Xingu até Conceição do Araguaia onde descia fluvialmente até Belém; e (iii) a partir dos anos 1950-60, após o declínio da economia da borracha, pela conclusão da rodovia Belém-Brasília, política dos governos militares de ocupação da Amazônia, início do processo de privatização das terras devolutas do Estado do Pará e crescimento da agricultura e pecuária (ibid).

Ao longo das décadas, houve o crescimento dos núcleos urbanos e, atualmente, o Araguaia Paraense é composto por quinze municípios: Água Azul do Norte, Bannach, Conceição do Araguaia, Cumaru do Norte, Floresta do Araguaia, Ourilândia do Norte, Pau D'arco, Redenção, Rio Maria, Santa Maria das Barreiras, Santana do Araguaia, São Félix do Xingu, Sapucaia, Tucumã e Xinguara. Assim, com o intuito de um olhar mais aproximado, este artigo visa analisar a evolução do Covid-

\footnotetext{
${ }^{1}$ Essa região também é denominada por Região de Integração Araguaia pelo Governo do Pará, por Região de Saúde Araguaia pelo Sistema Único de Saúde (SUS) e por Região Geográfica Intermediária de Redenção pelo Instituto Brasileiro de Geografia e Estatística (IBGE).
} 
19, parte das infraestruturas hospitalares e a hierarquia urbana nos municípios araguaianos paraenses. Para tal, são apresentados, a seguir, um referencial teórico, sua metodologia, os resultados encontrados, a discussão e as conclusões desta pesquisa.

\section{Referencial teórico}

Por se tratar de um caso pouco estudado, são apresentadas, nesta seção, investigações sobre infraestrutura hospitalar, Covid-19 e hierarquia urbana no Araguaia Paraense. Em um ano típico, sem a influência do novo coronavírus, Rache et al (2020) destacam que 279 das 436 regiões (64\%) no Brasil estão abaixo do mínimo desejado em relação a leitos de Unidade de Terapia Intensiva (UTI) totais (SUS e privados), sendo $316(72,48 \%)$ tratando-se somente do SUS. Vale ressaltar que 142 regiões não possuem qualquer leito, o que corresponde a $14,9 \%$ da população brasileira que depende exclusivamente do SUS, com maior proporção no Centro-Oeste (21\%), Nordeste $(30,5 \%)$ e Norte $(22,6 \%)$. Conforme Figura 2, o Araguaia Paraense encontra-se abaixo do mínimo desejável.

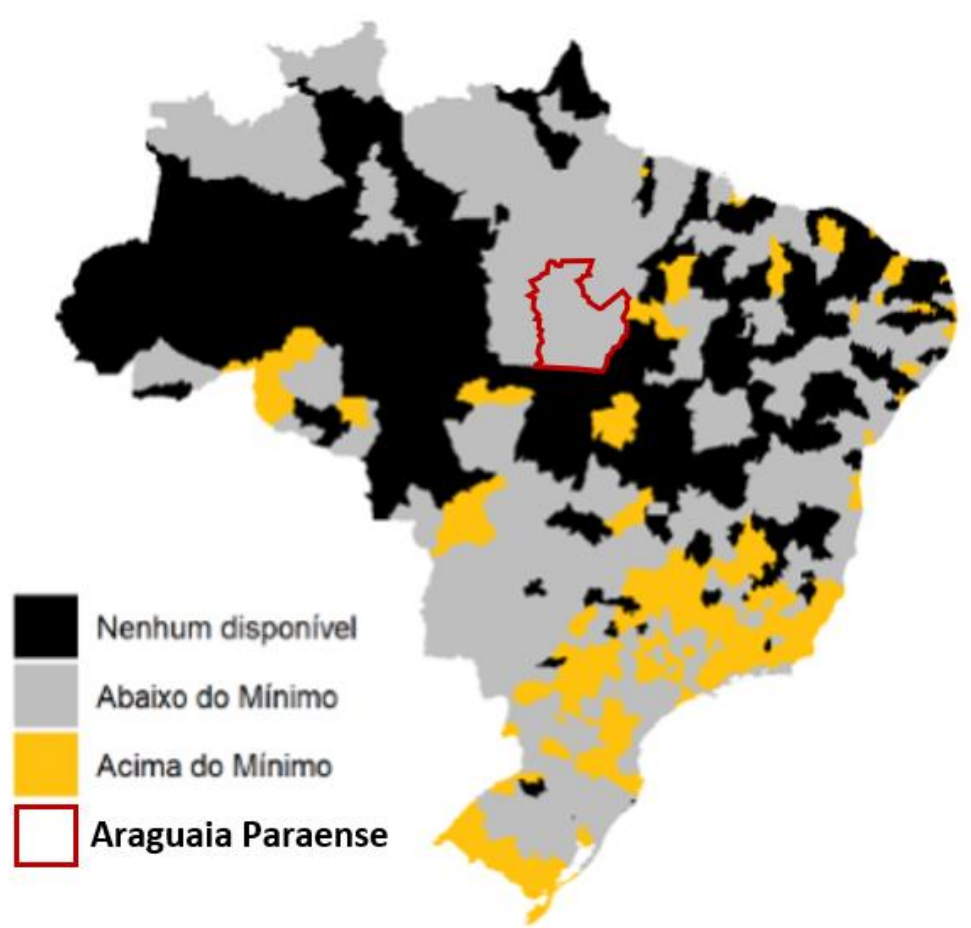

Figura 2 - Leitos de UTI do SUS por 100 mil habitantes

Notas: (1) Mínimo desejável de 10 leitos a cada 100 mil habitantes; (2) dados do Cadastro Nacional dos Estabelecimentos de Saúde (CNES); e (3) delimitação do Araguaia Paraense feita pelos autores. Fonte: Rache et al (2020, p. 3)

Sobre Covid-19, são poucas pesquisas no Araguaia Paraense. A Universidade Federal do Sul e Sudeste do Pará (Unifesspa) está monitorando os municípios onde tem campi no Sudeste Paraense, sendo que três situam-se no Araguaia Paraense: Santana do Araguaia, São Félix do Xingu e Xinguara. Com referência em agosto, destacam-se baixa taxa de isolamento social e aumento do registro de casos. Em Santana, os infectados são de maioria do sexo masculino (53\%), de cor parda (70\%) e com significativo aumento entre os profissionais da saúde. Em São Félix do Xingu, o maior percentual de afetados foi na fase mais economicamente ativa, em mulheres (53\%), em pardos $(62 \%)$ e em indígenas (22\%). Xinguara registrou maior percentual de mulheres (53\%) e mais de 100 novos casos diários de Covid-19 (UNIFESSPA, 2020).

Nessa região em estudo, embora seus municípios sejam estruturas urbanas políticoadministrativas independentes, é importante entender as relações de interdependência entre si 
(Villaça, 2001). Em relação aos arranjos populacionais ${ }^{2}$, a pesquisa Regiões de Influência das Cidades (Regic) do IBGE (2020a) classifica Redenção como Centro Sub-Regional A que começa "a estruturar sua própria rede [urbana]" (ibid, p. 14); Xinguara, Centro Sub-Regional B; Conceição do Araguaia, Centro de Zona A; e Tucumã, Centro de Zona $\mathrm{B}^{3}$. Essa área se vincula a três arranjos: Belém (Metrópole 1C), Goiânia (Metrópole 1C) e Palmas (Capital Regional B - 2B). No caso de Palmas, seu vínculo acontece de forma direta e somente com a cidade de Santana do Araguaia. Conforme Figuras 3 e 4, junto de Belém, "a influência de Goiânia é exercida diretamente sobre o Centro Sub-Regional de Redenção” (ibid, p. 16).

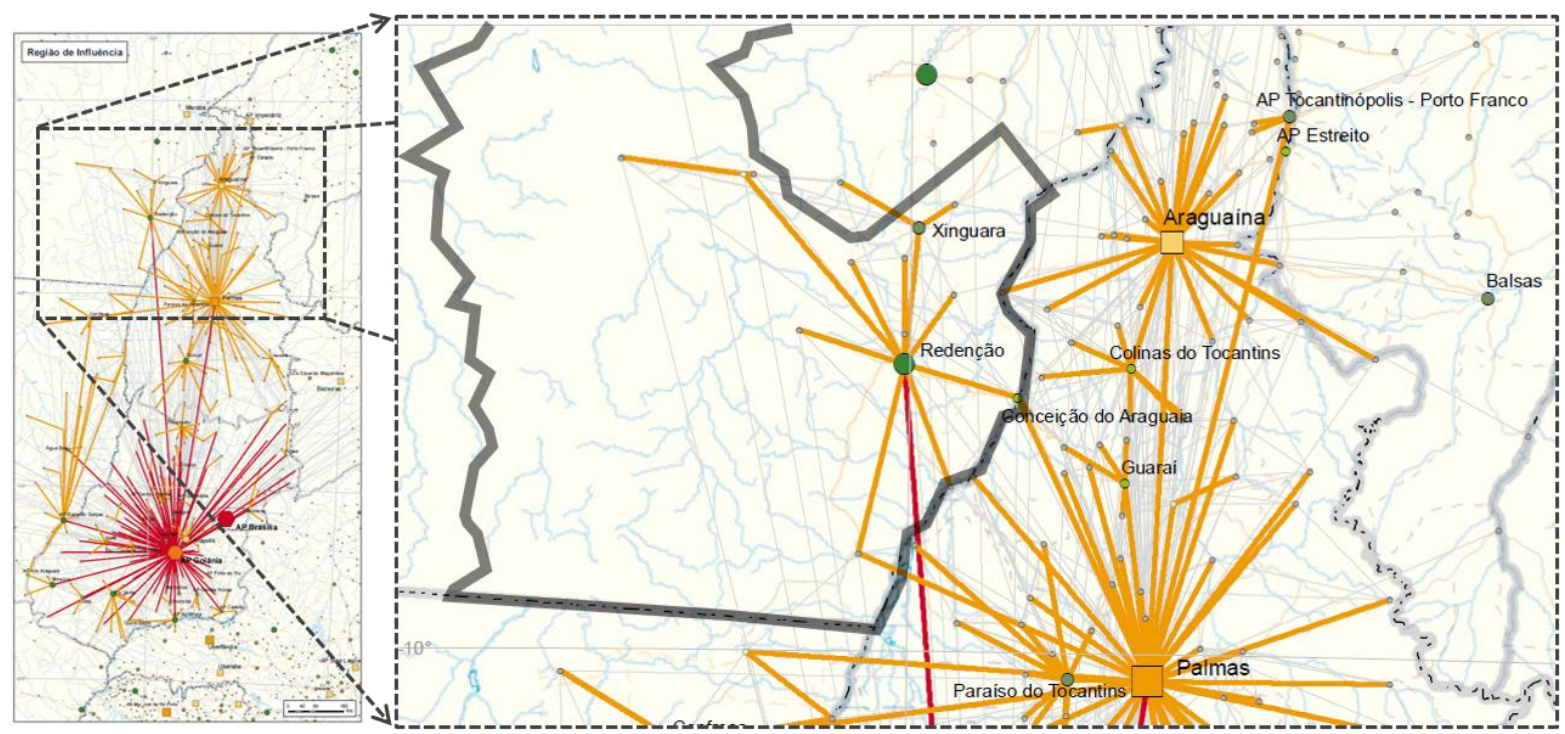

Figura 3 - Arranjo Populacional de Goiânia

Fonte: IBGE (2020a, p. 23) com adaptação dos autores.

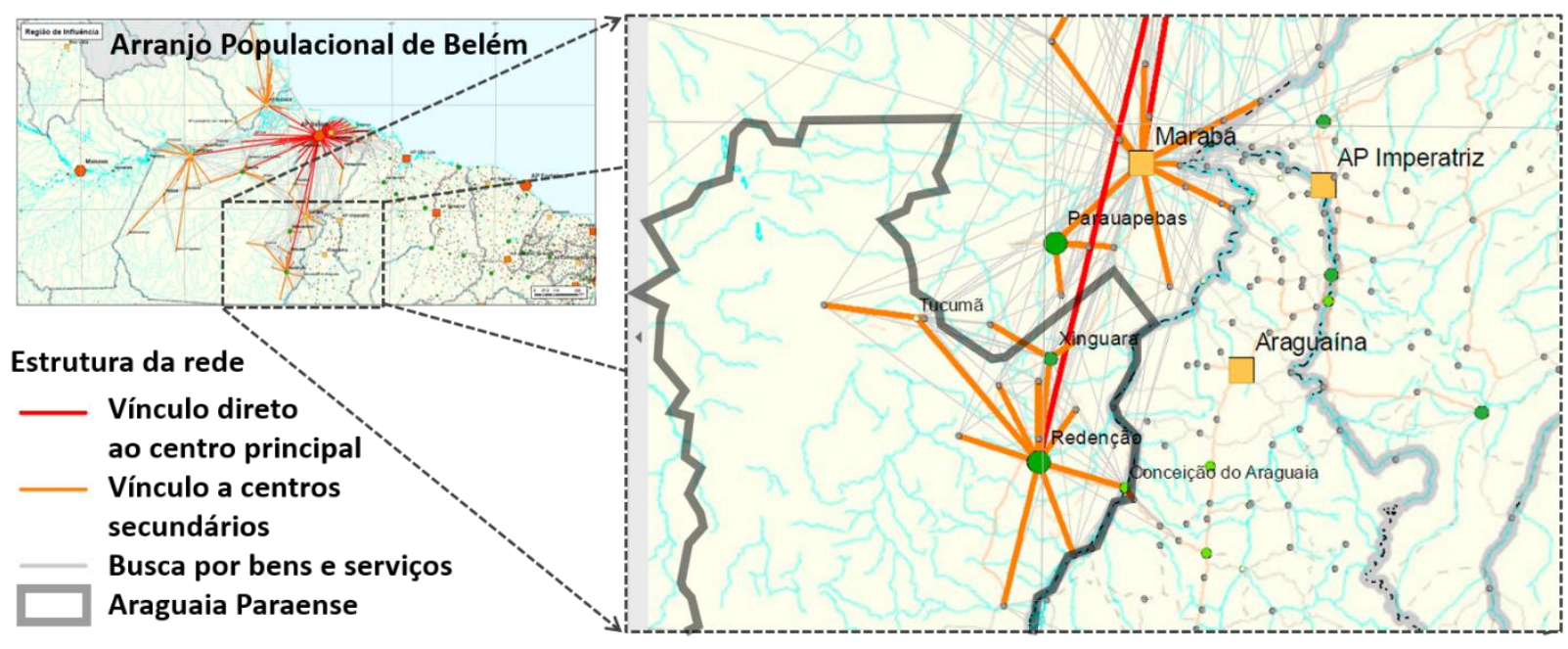

Figura 4 - Arranjo Populacional de Belém

Fonte: IBGE (2020a, p. 29) com adaptação dos autores.

Sobre deslocamentos para serviços de saúde, conforme Figura 5, são apresentadas duas categorias: de baixa e média complexidade, para consultas que não impliquem em internação; e alta

\footnotetext{
2 “Os Arranjos Populacionais são unidades territoriais compostas por mais de um Município, que apresentam integração significativa em razão da contiguidade das áreas urbanizadas ou da presença de deslocamentos frequentes dos habitantes para trabalhar ou estudar" (IBGE, 2020a, p. 72).

${ }^{3}$ Os centros urbanos são classificados como Metrópoles (Grande Metrópole Nacional, Metrópole Nacional e Metrópole), Capitais Regionais (A, B e C), Centros Sub-Regionais (A e B), Centros de Zona (A e B) e Centros Locais (quando sua influência se restringe em grande maioria aos seus próprios moradores) (IBGE, 2020a).
} 
complexidade, para "tratamentos especializados com alto custo, envolvendo: internação; cirurgias; ressonância magnética; tomografia; e tratamentos de câncer” (ibid, p. 111). Em ambos os casos, Redenção apresenta significativa importância para região, sendo que nos serviços de alta complexidade, há maior dependência com cidades de fora da região.
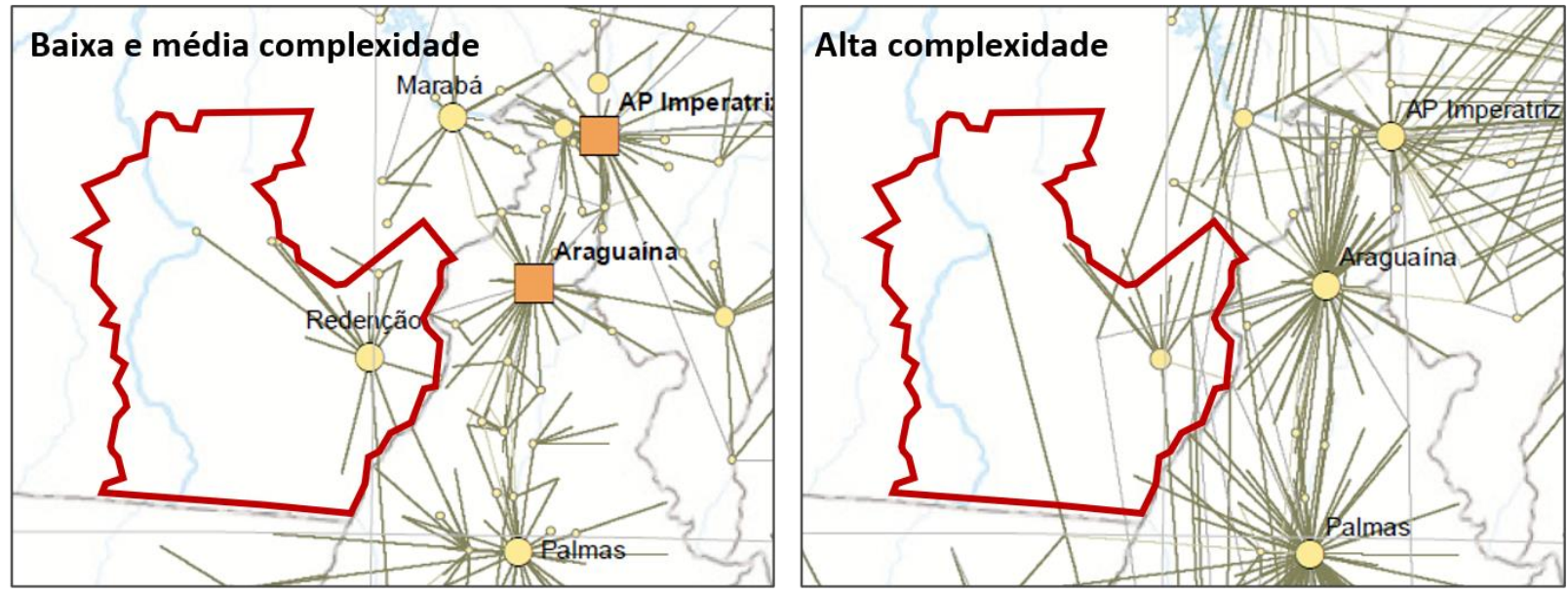

Araguaia Paraense

Figura 5 - Deslocamentos para serviços de saúde (2018)

Fonte: IBGE (2020a, p. 110, 112) com adaptação dos autores.

\section{Metodologia}

Para alcançar o objetivo proposto, esta pesquisa exploratória conta com coleta de dados secundários sobres questões demográficas, de leitos, de alguns equipamentos e de casos e óbitos por Covid-19. Os dados demográficos sobre municípios, Pará e Brasil foram obtidos no site do IBGE (2020b). Em relação aos leitos e alguns equipamentos hospitalares, foi consultado o site do SUS, cujos dados são referentes ao mês de agosto (DATASUS, 2020). Os dados sobre leitos complementares abrangem tanto UTI quanto unidade intermediária. Na pesquisa de Rache et al (2020), foi adotado como desejável 10 a 30 leitos de UTI por 100 mil habitantes. Por se tratar de municípios com menor população, foi considerada a proporção contrária do máximo desejável de 10 mil habitantes por leito. A Resolução $n^{\circ} 7$ (MS, 2010) recomenda o mínimo de um ventilador mecânico para cada dois leitos de UTI e uma reserva operacional de mais um equipamento a cada 5 leitos. Para facilitar a estimativa, foram excluídas a reserva operacional e as demais particularidades, considerado o máximo desejável de 20 mil habitantes por equipamento. Deve ser garantido também a tomografia computadorizada relacionada à UTI. Os casos e óbitos de Covid-19 registrados foram coletados no site oficial do governo federal, em 03 de outubro, sendo que não há números sobre recuperados (CB, 2020). Por fim, esses dados foram processados em tabelas e figuras chegando aos resultados apresentados a seguir

\section{O Araguaia Paraense, sua infraestrutura hospitalar e a Covid-19}

\subsection{Municípios araguaianos paraenses}

O Araguaia Paraense, conforme Tabela 1, abrange 13,88\% do território estadual e 2,03\% do nacional. Apesar do aparente baixo percentual, essa região é maior que os Estados do Acre ou do Ceará, por exemplos. Em relação aos habitantes, sua população de menos de 500 mil habitantes representa 5,68\% do Pará e 0,23\% do Brasil. 
Tabela 1 - Dados demográficos do Araguaia Paraense, Pará e Brasil

\begin{tabular}{|c|c|c|c|}
\hline Municípios & $\begin{array}{c}\text { Pop. estimada } \\
{[2020]}\end{array}$ & $\begin{array}{l}\text { Área da unid. ter. } \\
\text { [2019] }\left(\mathrm{km}^{2}\right)\end{array}$ & $\begin{array}{c}\text { Dens. Demog. } \\
(\text { hab./km²) }\end{array}$ \\
\hline Água Azul do Norte & 27.615 & $7.113,955$ & 3,88 \\
\hline Bannach & 3.262 & $2.956,649$ & 1,10 \\
\hline Conceição do Araguaia & 45.557 & $5.829,482$ & 7,81 \\
\hline Cumaru do Norte & 10.466 & $17.085,001$ & 0,61 \\
\hline Floresta do Araguaia & 17.768 & $3.444,285$ & 5,16 \\
\hline Ourilândia do Norte & 27.359 & $14.410,567$ & 1,90 \\
\hline Pau D'Arco & 6.033 & $1.671,419$ & 3,61 \\
\hline Redenção & 75.556 & $3.823,809$ & 19,76 \\
\hline Rio Maria & 17.697 & $4.114,627$ & 4,30 \\
\hline Santa Maria das Barreiras & 17.206 & $10.330,214$ & 1,67 \\
\hline Santana do Araguaia & 74.419 & $11.591,443$ & 6,42 \\
\hline São Félix do Xingu & 91.340 & $84.212,958$ & 1,08 \\
\hline Sapucaia & 5.047 & $1.298,190$ & 3,89 \\
\hline Tucumã & 33.690 & $1.298,190$ & 25,95 \\
\hline Xinguara & 40.573 & $3.779,348$ & 10,74 \\
\hline Araguaia (total) & 493.588 & $172.960,137$ & 2,85 \\
\hline Pará & 8.690 .745 & $1.245 .870,798$ & 6,98 \\
\hline Araguaia/Pará (\%) & $5,68 \%$ & $13,88 \%$ & - \\
\hline Brasil & 211.755 .692 & $8.510 .295,914$ & 24,88 \\
\hline Araguaia/Brasil (\%) & $0,23 \%$ & $2,03 \%$ & - \\
\hline
\end{tabular}

Fonte: Elaboração própria com dados do IBGE (2020b).

Sobre a extensão territorial, São Félix do Xingu é o maior município, cuja área equivale a 87,96\% do Estado de Santa Catarina. Depois estão Cumaru do Norte, Ourilândia do Norte, Santana do Araguaia, Santa Maria das Barreiras etc. As cinco cidades mais populosas são em ordem decrescente: São Félix do Xingu, Redenção, Santana do Araguaia, Conceição do Araguaia e Xinguara. Quase a metade dos municípios tem menos de 20 mil habitantes: Bannach, Cumaru do Norte, Floresta do Araguaia, Pau D’Arco, Rio Maria, Santa Maria das Barreiras e Sapucaia. Com maior densidade demográfica, estão em ordem decrescente Tucumã, Redenção, Xinguara, Conceição do Araguaia e Santana do Araguaia. Entretanto, a maioria é composta por cidades com baixa densidade demográfica e amplas unidades territoriais, como no caso de Cumaru do Norte com 0,61 de densidade demográfica ou de São Félix do Xingu que, apesar de ser a mais populosa, apresenta aproximadamente $1 \mathrm{hab} . / \mathrm{km}^{2}$. Assim, tanto a região quanto a maior parte dos municípios estão abaixo das médias de densidade demográfica estadual e nacional.

\subsection{Leitos, tomógrafos e respiradores}

A Tabela 2 apresenta os leitos de internação e leitos complementares (UTI e unidade intermediária) no Araguaia Paraense. Sobre os leitos de internação, todos os municípios são contemplados, sendo que 80,92\% é de responsabilidade do SUS. As proporções desses leitos em relação ao Pará $(7,20 \%)$ e ao Brasil $(0,25 \%)$ são pouco maiores do que a relação entre suas populações $(5,68 \%$ e $0,23 \%)$. No entanto, ao olhar por seus munícipios, Água Azul do Norte, Cumaru do Norte, Floresta do Araguaia, Santa Maria das Barreiras, Santana do Araguaia e São Félix do Xingu tem médias de habitantes por leito superior a estadual. O pior caso é em Santana do Araguaia com 1.181 habitantes para cada leito, ou seja, 108,65\% maior do que a média do Pará de 566. Sobre leitos complementares, são apenas 126 leitos complementares em todo Araguaia 
Paraense que resultam em uma média desejável de 3.917 habitantes por leito. No entanto, saltam aos olhos os municípios sem tal infraestrutura. Pois são apenas oito cidades que dispõem de UTI ou unidade intermediária, sendo que seis são servidas apenas pelo SUS e duas, Redenção e Conceição do Araguaia, que possuem leitos complementares não pertencentes ao SUS e, nesses casos, são a maioria. No máximo desejável de 10.000 habitantes por leito, estão Bannach, Conceição do Araguaia, Redenção, Rio Maria, Santa Maria das Barreiras e Xinguara.

Tabela 2 - Leitos no Araguaia Paraense, Pará e Brasil

\begin{tabular}{l|c|c|c|c|c|c}
\hline & \multicolumn{2}{|c|}{ Leitos de internação } & \multicolumn{3}{c}{ Leitos Complementares } \\
\hline Municípios & SUS & $\begin{array}{c}\text { Não } \\
\text { SUS }\end{array}$ & $\begin{array}{c}\text { Hab./ } \\
\text { leitos }\end{array}$ & SUS & $\begin{array}{c}\text { Não } \\
\text { SUS }\end{array}$ & $\begin{array}{c}\text { Hab./ } \\
\text { leitos }\end{array}$ \\
\hline Água Azul do Norte & 40 & - & 690 & - & - & - \\
\hline Bannach & 10 & - & 326 & 1 & - & 3.262 \\
\hline Conceição do Araguaia & 117 & 2 & 383 & 9 & 17 & 1.752 \\
\hline Cumaru do Norte & 11 & - & 951 & - & - & - \\
\hline Floresta do Araguaia & 20 & - & 888 & - & - & - \\
\hline Ourilândia do Norte & 65 & 2 & 408 & 1 & - & 27.359 \\
\hline Pau D'Arco & 22 & - & 274 & - & - & - \\
\hline Redenção & 187 & 91 & 272 & 25 & 37 & 1.219 \\
\hline Rio Maria & 31 & 25 & 316 & 4 & - & 4.424 \\
\hline Santa Maria das Barreiras & 23 & - & 748 & 5 & - & 3.441 \\
\hline Santana do Araguaia & 63 & - & 1.181 & - & - & - \\
\hline São Félix do Xingu & 98 & 47 & 630 & 2 & - & 45.670 \\
\hline Sapucaia & 14 & - & 361 & - & - & - \\
\hline Tucumã & 87 & 12 & 340 & - & - & - \\
\hline Xinguara & 107 & 32 & 292 & 25 & - & 1.623 \\
\hline Araguaia (total) & $\mathbf{8 9 5}$ & $\mathbf{2 1 1}$ & $\mathbf{4 4 6}$ & $\mathbf{7 2}$ & $\mathbf{5 4}$ & $\mathbf{3 . 9 1 7}$ \\
\hline Pará & 11.882 & 3.486 & 566 & 1.254 & 1.378 & 3.302 \\
\hline Araguaia/Pará (\%) & $7,53 \%$ & $6,05 \%$ & - & $5,74 \%$ & $3,92 \%$ & - \\
\hline Brasil & 317.022 & 132.919 & 471 & 44.239 & 43.738 & 2.407 \\
\hline Araguaia/Brasil (\%) & $0,28 \%$ & $0,16 \%$ & - & $0,16 \%$ & $0,12 \%$ & - \\
\hline Notas:(1) Referencia & & & & & - \\
\end{tabular}

Notas: (1) Referência de Agosto de 2020; (2) Em leitos de internação, são consideradas as categorias de leitos cirúrgicos, clínicos, obstétricos, pediátricos, hospital dia e outras especialidades, sem considerar os leitos de observação; e (3) Em leitos complementares, são consideradas as categorias de leitos complementares (UTI e Unidade Intermediária).

Fonte: Elaboração própria com dados do MS - CNES (DATASUS, 2020).

Conforme Tabela 3, o equipamento respirador/ventilador está presente em dois terços dos municípios da região, enquanto tomógrafo computadorizado em apenas um terço. 100\% dos tomógrafos estão em uso, enquanto respirador/ventilador, 92,05\%. Os municípios que dispõem dos dois equipamentos são Conceição do Araguaia, Ourilândia do Norte, Redenção, Tucumã e Xinguara. Considerando o desejável de um ventilador por 20 mil habitantes, somente Conceição do Araguaia, Cumaru do Norte, Floresta do Araguaia, Redenção, Santa Maria das Barreiras, Tucumã e Xinguara alcançam esta meta. Ao comparar com o Pará e o Brasil, o Araguaia apresenta uma média menor.

Tabela 3 - Tomógrafo e respirador no Araguaia Paraense, Pará e Brasil

\begin{tabular}{|l|l|} 
Tomóg. comp. & Respirador/ventilador \\
\hline
\end{tabular}




\begin{tabular}{l|l|l|l|l|l|l} 
Municípios & $\begin{array}{l}\text { Estab. do } \\
\text { SUS }\end{array}$ & $\begin{array}{l}\text { Equip. } \\
\text { exist. }\end{array}$ & $\begin{array}{l}\text { Estab. } \\
\text { do SUS }\end{array}$ & $\begin{array}{l}\text { Equip. } \\
\text { em uso }\end{array}$ & $\begin{array}{l}\text { Equip. } \\
\text { exist. }\end{array}$ & $\begin{array}{l}\text { Hab./ } \\
\text { equip. }\end{array}$ \\
\hline Conceição do Araguaia & 2 & 2 & 2 & 6 & 6 & 7.593 \\
\hline Cumaru do Norte & - & - & 1 & 1 & 1 & 10.466 \\
\hline Floresta do Araguaia & - & - & 1 & - & 1 & 17.768 \\
\hline Ourilândia do Norte & 1 & 1 & 1 & 1 & 1 & 27.359 \\
\hline Redenção & 1 & 2 & 2 & 57 & 62 & 1.219 \\
\hline Santa Maria das Barreiras & - & - & 2 & 2 & 2 & 8.603 \\
\hline Santana do Araguaia & - & - & 1 & 1 & 1 & 74.419 \\
\hline São Félix do Xingu & - & - & 2 & 3 & 3 & 30.447 \\
\hline Tucumã & 2 & 2 & 4 & 4 & 4 & 8.423 \\
\hline Xinguara & 1 & 1 & 2 & 6 & 7 & 5.796 \\
\hline Araguaia (total) & $\mathbf{7}$ & $\mathbf{8}$ & $\mathbf{1 8}$ & $\mathbf{8 1}$ & $\mathbf{8 8}$ & $\mathbf{5 . 6 0 9}$ \\
\hline Pará & 74 & 140 & 193 & 1.775 & 1.883 & 4.615 \\
\hline Araguaia/Pará & $9,46 \%$ & $5,71 \%$ & $9,33 \%$ & $4,56 \%$ & $4,67 \%$ & - \\
\hline Brasil & 2.315 & 5.336 & 6.577 & 77.539 & 81.778 & 2.589 \\
\hline Araguaia/BR & $0,30 \%$ & $0,15 \%$ & $0,27 \%$ & $0,10 \%$ & $0,11 \%$ & - \\
\hline Nota:(1) Refer
\end{tabular}

Nota: (1) Referência de Agosto de 2020; e (2) Por não dispor dos equipamentos listados, foram excluídos desta Tabela os municípios Água Azul do Norte, Bannach, Pau D'Arco, Rio Maria e Sapucaia.

Fonte: Elaboração própria com dados do MS - CNES do Brasil (DATASUS, 2020).

\subsection{Casos e óbitos registrados de Covid-19}

Os primeiros casos registrados na Região do Araguaia foram em Conceição do Araguaia e Tucumã em 14 de abril de 2020 e Santa Maria das Barreiras foi a última cidade a registrar o primeiro caso em 22 de maio. Conforme Figura 6, é possível observar Redenção com maior aceleração e registro de toda a região com quase 4 mil casos. Entre 2 e 3 mil casos, estão na ordem decrescente Ourilândia do Norte, Tucumã, Xinguara e São Félix do Xingu; e, entre 1 e 2 mil, Conceição do Araguaia e Santana do Araguaia. Mais da metade dos demais municípios encontram-se abaixo de mil registros.

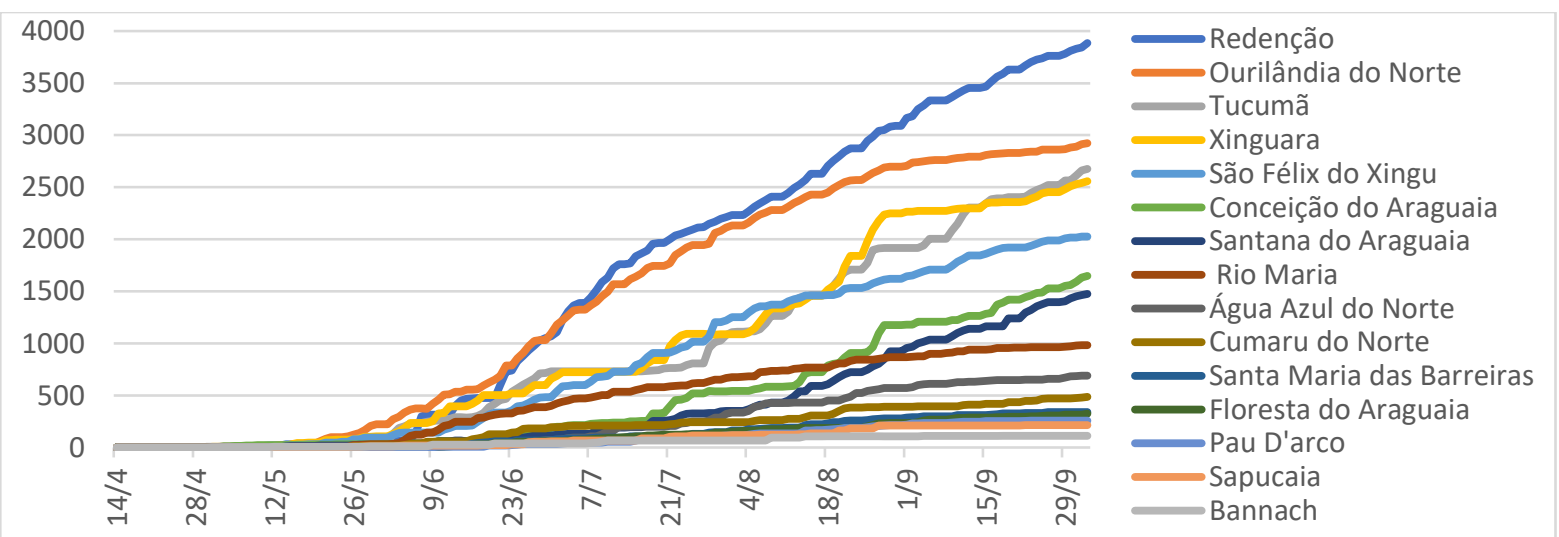

Figura 6 - Casos acumulados/registrados de Covid-19 nos municípios do Araguaia Paraense Fonte: Elaboração própria com dados obtidos em CB (2020).

A Figura 7 apresenta os casos diários de todo Araguaia Paraense e a média móvel de 7 dias. É possível observar o maior crescimento em dois momentos no mês junho quando a média móvel chega próxima aos 150 e aos 250 casos diários; uma pequena diminuição com estabilização entre 150 e 200 casos nos meses de julho e início de agosto; um novo pico de 300 casos no final de agosto; 
e diminuição e estabilidade no mês de setembro com média ligeiramente acima de 100 casos por dia.

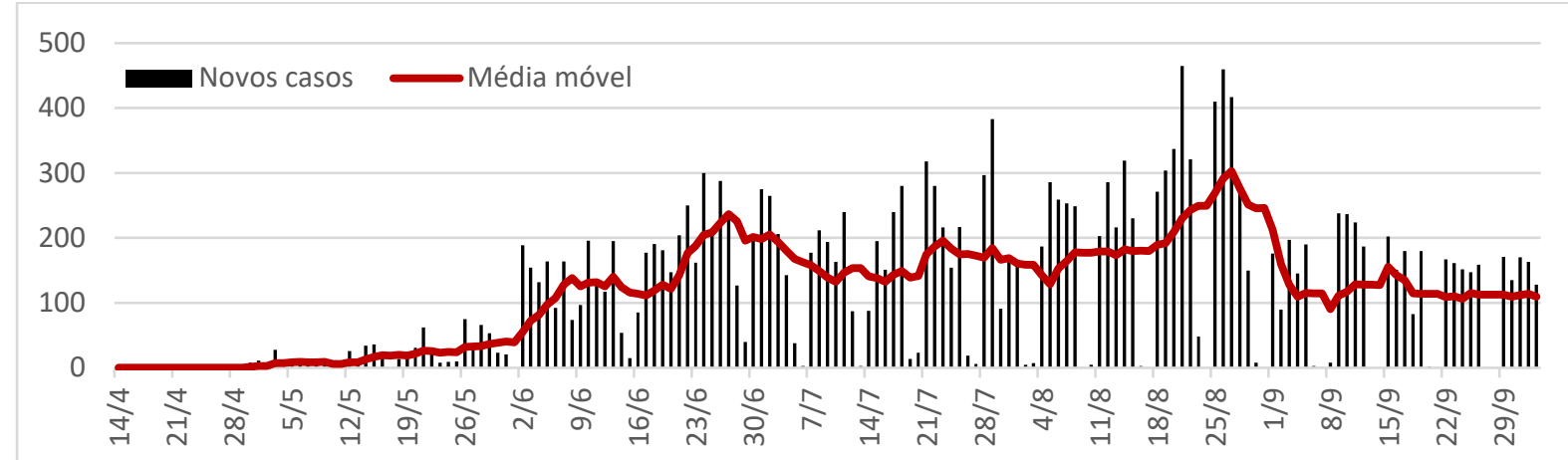

Figura 7 - Média móvel ( 7 dias) de casos de Covid-19 registrados por dia

Fonte: Elaboração própria com dados obtidos em CB (2020).

Em relação aos óbitos pelo novo coronavírus, conforme Figura 8, Redenção é também o município com maior registro, 55, seguidos por Tucumã com 31, Xinguara com 26, Conceição do Araguaia com 20 e São Félix do Xingu com 16.

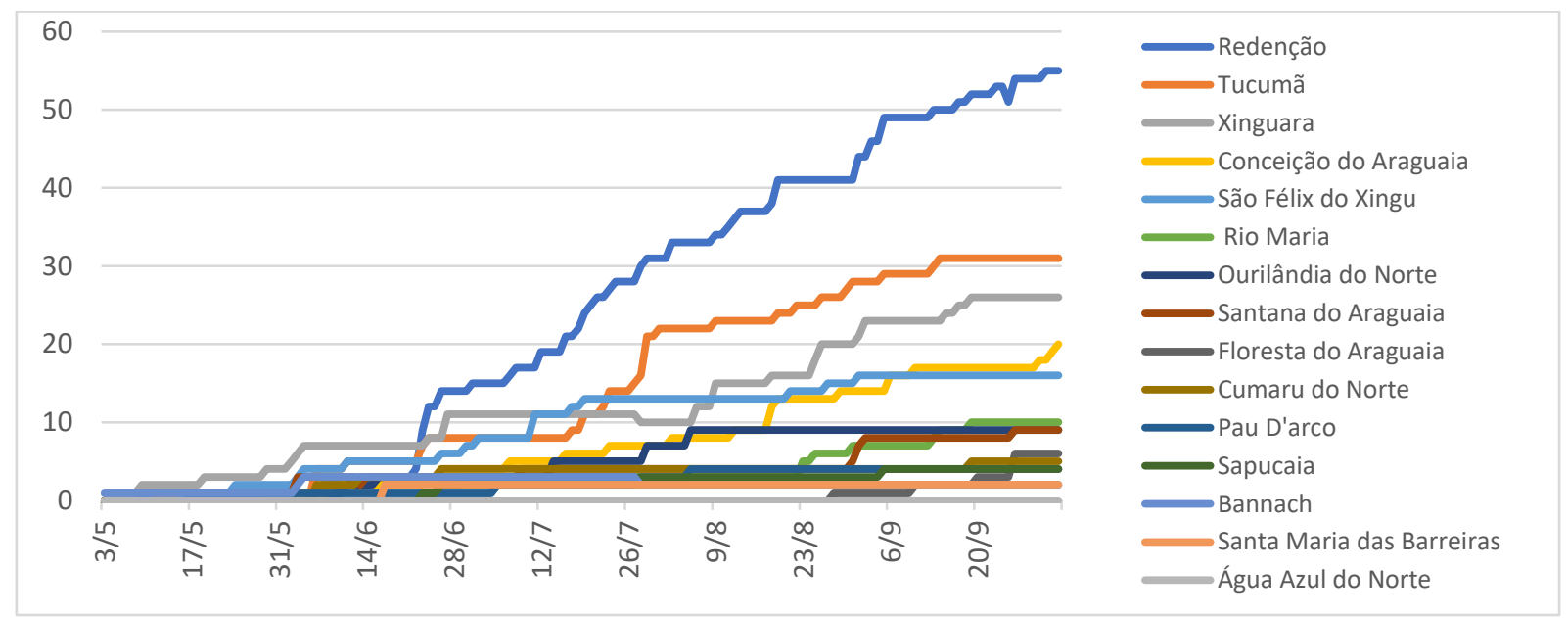

Figura 8 - Óbitos acumulados/registrados por Covid-19 nos municípios do Araguaia Paraense

Fonte: Elaboração própria com dados obtidos em CB (2020).

Em relação à média móvel de 7 dias do Araguaia Paraense, são observados alguns picos de 2 e 4 mortes diárias em junho, uma variação entre 1 e 2 em julho e agosto, novo pico de 3 no início de setembro e uma variação em torno de 1 óbito diário no restante do mês (ver Figura 9). 


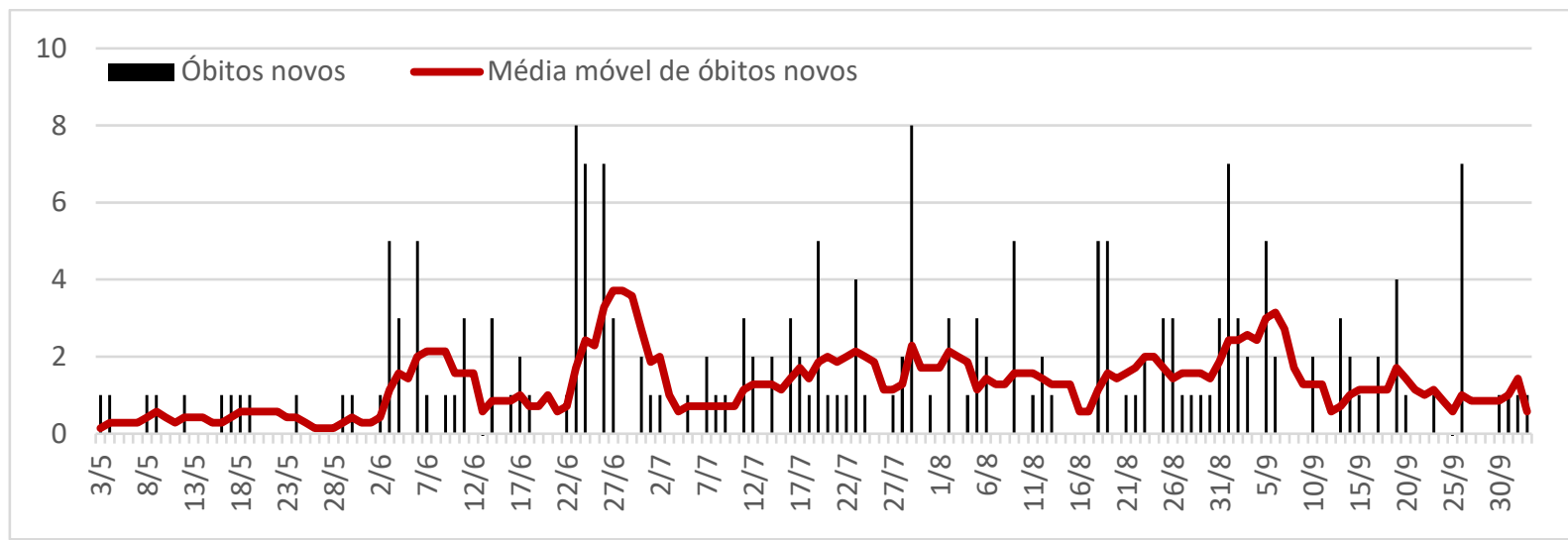

Figura 9 - Média móvel (7 dias) de óbitos de Covid-19 registrados por dia

Fonte: Elaboração própria com dados obtidos em CB (2020).

De acordo com a Tabela 4, o Araguaia Paraense alcançou, em 03 de outubro, o total acumulado de 20.600 casos e 199 óbitos por Covid-19. Floresta do Araguaia tem a menor taxa de contaminação, 1.829,1 casos a cada 100 mil habitantes, e com a maior taxa está Ourilândia do Norte com 10.683,9 casos a cada 100 mil habitantes. Santa Maria das Barreiras tem a menor taxa de mortalidade, 11,6 óbitos a cada 100 mil habitantes, e Ourilândia do Norte tem a menor taxa de letalidade, $0,30 \%$. Tucumã apresenta a maior taxa de mortalidade, 92 óbitos a cada 100 mil habitantes, e Sapucaia, a maior taxa de letalidade, 1,86\%. Ao comparar com o Pará e o Brasil, essa região apresenta, por um lado, uma maior taxa de contaminação e, por outro, menores taxas de mortalidade e de letalidade. Com alta contaminação, destacam-se os municípios de Bannach, Conceição do Araguaia, Cumaru do Norte, Ourilândia do Norte, Pau D’Arco, Redenção, Rio Maria, Sapucaia, Tucumã e Xinguara que alcançaram taxas maiores que a estadual. Em relação à mortalidade, Redenção, Sapucaia e Tucumã apresentam taxas superiores à nacional.

Tabela 4 - Casos e óbitos registrados por Covid-19, Taxa de Contaminação (TC), Taxa de Mortalidade e Taxa de Letalidade (LT) na Região do Araguaia, no Pará e no Brasil

\begin{tabular}{|c|c|c|c|c|c|}
\hline Municípios & $\begin{array}{l}\text { Casos } \\
\text { acum. }\end{array}$ & $\begin{array}{l}\text { TC (casos/ } \\
\text { 100mil hab.) }\end{array}$ & $\begin{array}{l}\text { Óbitos } \\
\text { acum. }\end{array}$ & $\begin{array}{l}\text { TM (óbitos/ } \\
\text { 100mil hab.) }\end{array}$ & $\begin{array}{l}\text { LT (óbitos/ } \\
\text { casos) }\end{array}$ \\
\hline Água A. do Norte & 690 & $2.498,6$ & 0 & 0,0 & - \\
\hline Bannach & 112 & $3.433,5$ & 2 & 61,3 & $1,79 \%$ \\
\hline Conceição do Ar. & 1.648 & $3.617,4$ & 20 & 43,9 & $1,21 \%$ \\
\hline Cumaru do Norte & 486 & $4.643,6$ & 5 & 47,8 & $1,03 \%$ \\
\hline Floresta do Araguaia & 325 & $1.829,1$ & 6 & 33,8 & $1,85 \%$ \\
\hline Ourilândia do N. & 2.923 & $10.683,9$ & 9 & 32,9 & $0,30 \%$ \\
\hline Pau D'Arco & 258 & $4.276,5$ & 4 & 66,3 & $1,55 \%$ \\
\hline Redenção & 3.885 & $5.141,9$ & 55 & 72,8 & $1,42 \%$ \\
\hline Rio Maria & 983 & $5.554,6$ & 10 & 56,5 & $1,02 \%$ \\
\hline S. M. Barreiras & 340 & $1.976,1$ & 2 & 11,6 & $0,56 \%$ \\
\hline Santana do Ar. & 1.475 & $1.982,0$ & 9 & 12,1 & $0,61 \%$ \\
\hline S. Félix do Xingu & 2.026 & $2.218,1$ & 16 & 17,5 & $0,79 \%$ \\
\hline Sapucaia & 215 & $4.260,0$ & 4 & 79,3 & $1,86 \%$ \\
\hline Tucumã & 2.676 & $7.943,0$ & 31 & 92,0 & $1,16 \%$ \\
\hline Xinguara & 2.558 & $6.304,7$ & 26 & 64,1 & $1,01 \%$ \\
\hline Araguaia (total) & 20.600 & $4.173,5$ & 199 & 40,3 & $0,97 \%$ \\
\hline Pará & 233.802 & $2.690,2$ & 6.593 & 75,9 & $2,82 \%$ \\
\hline Brasil & 4.906 .833 & $2.317,2$ & 145.987 & 68,9 & $2,98 \%$ \\
\hline
\end{tabular}

Nota: Dados de Covid-19 de 03 de outubro de 2020

Fonte: Elaboração própria com dados de Covid-19 do CB (2020) e de população do IBGE (2020b). 


\section{Discussões}

Por se tratar de uma questão tão recente, é importante o desenvolvimento de novas pesquisas sobre Covid-19 nas suas diversas áreas, incluindo nos campos multidisciplinares do Planejamento Urbano e Regional. Assim, esta pesquisa se dedica ao caso pouco estudado da Região do Araguaia Paraense, da relação entre seus munícipios, de parte de suas infraestruturas hospitalares e da evolução dos casos registrados do novo coronavírus. O desenho de pesquisa permite gerar uma fotografia sobre parte desta realidade, embora seja importante ressaltar fragilidades pela subnotificação de casos e a necessidade de ampliação da coleta de dados. De qualquer modo, com os dados disponíveis, é possível observar o aumento da média móvel de casos de Covid-19 em agosto, condizente com relatório da Unifesspa (2020). Entretanto, em setembro, observa-se uma diminuição com estabilidade ligeiramente acima de 100 casos por dia e variações próximas a um óbito diário.

Sobre a infraestrutura hospitalar, destaca-se que a média de equipamentos por habitante no Pará já é discrepante em relação ao Brasil, sendo que o Araguaia é maior ainda. Ao comparar com os parâmetros de máximo desejável de 10 mil habitantes por leito de UTI (complementar) (Rache et al, 2020) e de 20 mil habitantes por ventilador (MS, 2010), os números da região não são alarmantes. No entanto, estão em grande parte concentrados em poucas cidades, como Redenção, o Centro Sub-Regional A. Ademais, se trata de uma região com baixa densidade demográfica, núcleos urbanos distantes entre si e conectados em alguns casos por estradas precárias, incluindo pontes de madeiras vulneráveis tanto nas épocas de seca, pela queimada, quanto de chuva, com as enchentes. Logo, essas médias podem demonstrar uma aparente equidade quando na verdade muitos habitantes estão sem a garantia do direito à saúde. Isso pode ser observado nos dados municipais, tal como em Santana do Araguaia cuja média 1.181 habitantes para cada leito de internação é mais que o dobro da média do Pará (566 hab./leito) e não há leito complementar.

A Figura 10 permite observar no espaço a distribuição dos leitos complementares e a Taxa de Contaminação.
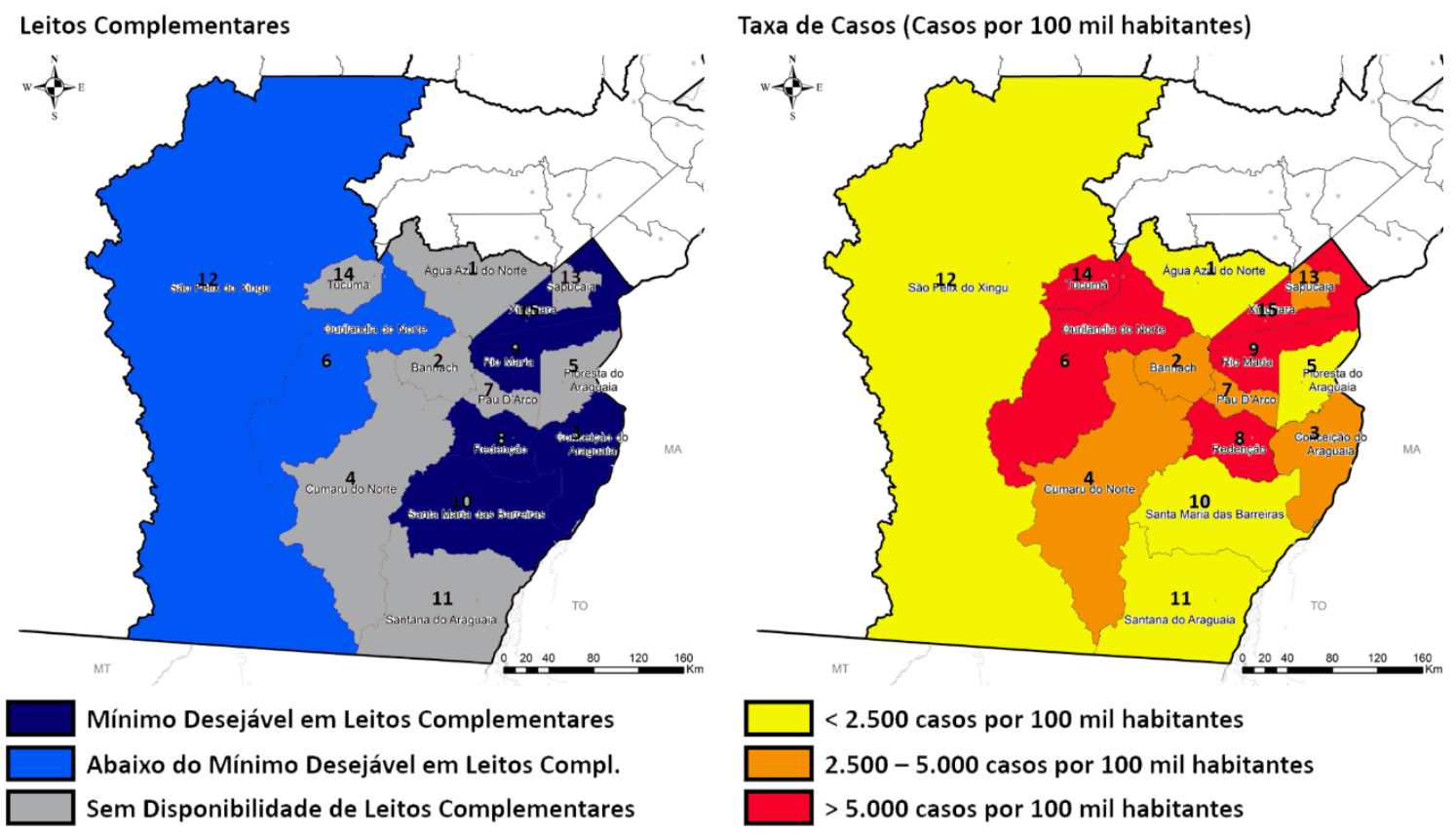

Municípios: (1) Água Azul do Norte, (2) Bannach, (3) Conceição do Araguaia, (4) Cumaru do Norte, (5) Floresta do Araguaia, (6) Ourilândia do Norte, (7) Pau D’arco, (8) Redenção, (9) Rio Maria, (10) Santa Maria das Barreiras, (11) Santana do Araguaia, (12) São Félix do Xingu, (13) Sapucaia, (14) Tucumã e (15) Xinguara

Figura 10 - Leitos Complementares e de Taxa de Contaminação no Araguaia Paraense

Fonte: Elaboração própria com dados de Covid-19 do CB (2020), de população do IBGE (2020b) e de infraestrutura hospitalar do MS - CNES do Brasil (DATASUS, 2020). 
Os Centros Sub-Regionais A, Redenção, e B, Xinguara, e o Centro de Zona A, Conceição do Araguaia, apresentam maiores infraestruturas e maiores registros de casos e óbitos. Das cidades menores, Ourilândia do Norte e Rio Maria também possuem maior infraestrutura e apresentam maiores taxas de contaminação. Por outro lado, São Félix do Xingu e Santana do Araguaia, respectivamente as primeira e terceira cidades mais populosas do Araguaia Paraense apresentam pouca infraestrutura e baixo registro de casos.

No ano típico de 2018, sem influência do Covid-19, a investigação do IBGE (2020) revela importante dependência dos municípios com Redenção. O cruzamento de todas essas informações levanta, por um lado, uma possível subnotificação nesses municípios mais populosos sem infraestrutura e, por outro, um indício que Redenção e outros municípios estejam exercendo forte vínculo em relação aos deslocamentos para serviços de saúde, nesse caso, por causa do novo coronavírus.

\section{Conclusões}

Esta pesquisa permite contribuir para o maior conhecimento do Araguaia Paraense no que tange à relação entre seus municípios, sua infraestrutura hospitalar e à propagação do Covid-19. Constata-se sua realidade periférica dentro do Estado do Pará onde muitos habitantes estão à margem do direito à saúde. Embora seja insuficiente, o serviço público ainda é o maior responsável pela garantia dos serviços. O SUS cobre mais de $80 \%$ dos leitos de internação que está presente em todas as cidades. No caso de leitos complementares, seis municípios só dispõem do SUS, enquanto somente Redenção e Conceição do Araguaia possuem leitos complementares não pertencentes ao SUS e, nesses casos, são a maioria. Em relação aos equipamentos, o SUS dispõe de respirador/ventilador em dois terços dos municípios da região, enquanto tomógrafo computadorizado em apenas um terço. Assim, entende-se necessário ampliar essa infraestrutura, sendo o serviço público com maior viabilidade do que a iniciativa privada.

Sobre os casos de Covid-19, a Região do Araguaia Paraense apresenta, por um lado, uma maior taxa de contaminação e, por outro, menores taxas de mortalidade e de letalidade. Isso pode ser um indicio de subnotificação a ser melhor investigado. A média móvel em setembro está acima de 100 casos por dia e variações próximas a um óbito diário. Além disso, é contrastante a diferença do registro de casos entre os municípios. Enquanto Redenção, Xinguara e Conceição do Araguaia, respectivamente as segunda, quarta e quinta cidades mais populosas, apresentam maiores infraestruturas e maiores registros de casos e óbitos, São Félix do Xingu e Santana do Araguaia, respectivamente as primeira e terceira cidades mais populosas não dispõem de infraestruturas e têm menor registro. Com isso, observa-se que as cidades já identificadas pelo IBGE como centros subregionais e centro de zona, sobretudo Redenção, podem estar desempenhando importante papel na hierarquia urbana e atraindo deslocamentos para serviços de saúde, nesse caso também para atendimento para casos do novo coronavírus. Como oportunidade de futuras investigações, acreditase que esse maior registro de casos em municípios com maior infraestrutura pode ser de habitantes de outras cidades com menor infraestrutura, além da possibilidade de subnotificação em municípios populosos com poucos registros. Isso pode contribuir ainda mais para entender as relações entre esses municípios, suas interdependências de infraestruturas e possibilidades de melhor desenvolvimento urbano e regional.

\section{Referências}

CB. Coronavírus Brasil. Painel Coronavírus. Atualizado em: 23/08/2020 18:00. Disponível em: <https://covid.saude.gov.br/>. Acesso em 03 out. 2020.

DATASUS. Disponível em: <https://datasus.saude.gov.br/cnes-recursos-fisicos/>. Acesso em 10 out. 2020.

IBGE. Instituto Brasileiro de Geografia e Estatística. (2020a). Regiões de influência das cidades: 2018 / IBGE, Coordenação de Geografia. IBGE. 
IBGE. Instituto Brasileiro de Geografia e Estatística. Disponível em: <https://cidades.ibge.gov.br/>. Acesso em 11 out. 2020b.

MS. Ministério da Saúde. Portaria n ${ }^{\circ} 188$, de 3 de fevereiro de 2020. Declara Emergência em Saúde Pública de importância Nacional (ESPIN) em decorrência da Infecção Humana pelo novo Coronavírus (2019-nCoV). Diário Oficial da União. Brasília, DF, 03 fev. 2020. Disponível em: https://www.in.gov.br/web/dou/-/portaria-n-188-de-3-de-fevereiro-de-2020-241408388. Acesso em 31 ago. 2020(b).

OPAS. Organização Pan-Americana de Saúde. Covid19. Disponível em: <https://www.paho.org/pt/covid19>. Acesso em 23 ago. 2020.

Rache, B., Rocha, R., Nunes, L., Spinola, P., Malik, A. M., \& Massuda, A. (2020). Necessidades de Infraestrutura do SUS em Preparo à COVID-19: Leitos de UTI, Respiradores e Ocupação Hospitalar. Nota Técnica n.3. IEPS: http://www.epsjv.fiocruz.br/sites/default/files/files/NT3\%20vFinal.pdf

Silva, F. C. A (2007). Economia Pastoril e os primórdios do capitalismo na Região do Araguaia paraense. 1890-1960. Novos Cadernos NAEA, v. 10, p. 5-22. https://periodicos.ufpa.br/index.php/ncn/article/view/68/120

UNIFESSPA. Universidade Federal do Sul e Sudeste do Pará. (2020). Panorama sobre a Covid-19 no sul e sudeste do Pará. Unifesspa.

Villaça, F. (2011). Espaço Intra-Urbano no Brasil (2 ed.). Studio Nobel. 